\title{
Exames de mamografia em Mato Grosso do Sul: análise da cobertura como componente de equidade*
}

\author{
Emiliana Akiko Kohatsu** \\ Ana Rita Barbieri*** \\ Virginia Alonso Hortale****
}

SumÁrio: 1. Introdução; 2. Metodologia; 3. Resultados; 4. Discussão; 5. Conclusões.

Summary: 1. Introduction; 2. Methodology; 3. Results; 4. Discussion; 5. Conclusions.

Palavras-chave: mamografia; cobertura de serviços de saúde; equidade de acesso.

KEY WORDS: mammogram; health services coverage; access equity.

Este artigo analisa a cobertura da mamografia em Mato Grosso do Sul em 2004, considerando sua variação nas regiões e nos municípios que referenciam e realizam o exame. Para tanto, foi desenvolvido um estudo utilizando dados secundários como fontes de informações referentes ao ano de 2004. Foram analisados os 77 municípios do estado de Mato Grosso do Sul. A coleta dos dados e sua análise obedeceram à seguinte classificação: municípios sem serviços de mamografia que encaminham as mulheres para o exame; municípios com serviços de mamografia (municípios-referência) que realizam os exames. A análise dos resultados foi feita sobre as três regiões do estado. Os resultados apontaram: a oferta de exames é inferior ao necessário; a cobertura é desigual para as diferentes regiões; os termos de compromisso pactuados na PPI não atendem às recomendações do Consenso para Controle do Câncer de

\footnotetext{
* Artigo recebido em abr. 2008 e aceito em dez. 2008.

** Especialista em políticas e gestão em saúde. Auditora da Secretaria Estadual de Saúde de Mato Grosso do Sul. Endereço: Av. Afonso Pena, 3.547 - CEP 79002-072, Campo Grande, MS, Brasil. E-mail: ekohatsu@saude.ms.gov.br.

*** Doutora em ciências da saúde. Professora da Universidade Federal de Mato Grosso do Sul. Endereço: Unidade IX. UFMS. Cidade Universitária, s/nº - CEP 79070-900, Campo Grande, MS, Brasil.E-mail: anabarbi@terra.com.br.

***** Doutora. Pesquisadora da Escola Nacional de Saúde Pública Sérgio Arouca, Fundação Oswaldo Cruz. Endereço: Escola Nacional de Saúde Pública Sergio Arouca (Ensp). Rua Leopoldo Bulhões, 1.480 - Manguinhos - CEP 21041-210, Rio de Janeiro, RJ, Brasil. E-mail: virginia@ ensp.fiocruz.br.
} 
Mama. Foi possível concluir que somente em uma região a cobertura dos exames é homogênea. A região que tem maior concentração de mamógrafos apresenta acentuada desigualdade de cobertura.

Mammograms in Mato Grosso do Sul: an analysis of coverage as an equity factor

This article analyzes mammogram coverage in the state of Mato Grosso do Sul, Brazil, in 2004, considering the differences between the regions and towns that prescribe and those that carry out the exam. The study used secondary data from all of the state's 77 municipalities. Data collection and analysis had the following classification: towns that don't have mammogram services and refer the women to another location to have their exams; towns that have mammogram services (reference towns) and carry out the exam. All three regions of the state were analyzed. The results show that: the offer is below the demand for the exam; the coverage is unequal for the different regions; the commitment to the terms of the Programmed and Integrated Pact fulfill the recommendations of the Breast Cancer Control Consensus. In only one region the exam coverage is uniform. The region with the highest concentration of mammogram machines has very unequal coverage.

\section{Introdução}

No Brasil, o câncer de mama é a maior causa de óbitos por câncer na população feminina na faixa etária entre 40 e 69 anos. Nas últimas décadas, a taxa bruta de mortalidade por câncer de mama apresentou uma variação percentual de $76 \%$, passando de 5,77 em 1979 para 10,15 mortes por 100 mil mulheres em 2002. As estimativas de incidência, referentes ao ano de 2006, indicaram 48.930 casos novos de câncer de mama no país (Brasil, Ministério da Saúde, Instituto Nacional do Câncer, 2005).

O diagnóstico em fases adiantadas da doença, principalmente nos estágios III e IV, leva ao comprometimento dos resultados do tratamento e é considerado uma das principais dificuldades na redução da mortalidade.

O Programa Nacional de Controle do Câncer do Colo do Útero e de Mama - Viva Mulher - preconiza, de acordo com as recomendações do Consenso para Controle do Câncer de Mama e como parte do atendimento integral à mulher, a realização do exame clínico das mamas para mulheres de todas as faixas etárias. Para mulheres acima de 40 anos de idade, recomenda a realização anual do exame clínico e mamografia. O consenso recomenda que as mulheres tenham acesso garantido aos procedimentos de investi- 
gação diagnóstica e de tratamento quando necessário (Brasil, Ministério da Saúde, Instituto Nacional do Câncer, 2005; Brasil, Ministério da Saúde, 2004; Brasil, Ministério da Saúde, 2005)

Do ponto de vista da gestão do sistema de saúde, o exame da mamografia é um procedimento remunerado, incluído como de média complexidade, nível III na tabela de procedimentos do Sistema Único de Saúde, tendo em vista o cumprimento da Pactuação Programada e Integrada (PPI) e o Pacto pela Saúde (Brasil, Ministério da Saúde, Secretaria de Assistência à Saúde, 2006; Ministério da Saúde, 2006). O Plano Diretor de Regionalização (PDR) e a Programação Pactuada Integrada (PPI) são instrumentos de gestão propostos em 2002 com a edição da Norma Operacional de Assistência à Saúde (Noas) visando à organização das redes assistenciais no âmbito dos estados. Entre outras finalidades, garantem a equidade no acesso da população às ações e serviços de saúde, bem como a regulação do sistema de saúde no que diz respeito à média e alta complexidade hospitalar e ambulatorial (Mato Grosso do Sul, 2005; Mato Grosso do Sul, Secretaria de Estado de Saúde, 2002).

Não é atual a preocupação em tornar o conceito de equidade exequível. Viana (2003) observa que desde o final da década de 1980 a reforma implementada no país trouxe a noção de equidade como uma das questões de fundo, refletindo a estratégia formulada pela OMS "Saúde para todos no ano 2000", cuja proposta era de promover ações de saúde baseadas na noção de necessidade, destinadas a todos, independentemente de raça, gênero, condições sociais, entre outras diferenças. Para Tobar (2003) e Whitehead (2000), a noção de equidade surge porque, idealmente, todos deveriam ter a justa oportunidade de obter seu pleno potencial de saúde, de modo a não deixar ninguém em desvantagem para alcançá-lo, se isso puder ser evitado.

Travassos e colaboradores (2000) e Lucchese (2003) relatam estudos realizados no Brasil na década de 1990 que evidenciam a persistência de iniquidades, principalmente relacionadas à distribuição geográfica dos recursos humanos e equipamentos e ao acesso e utilização dos serviços, além das desigualdades sociais e econômicas das diferentes populações, ou seja, somente a igualdade no acesso e uso dos serviços de saúde não é suficiente para diminuir as desigualdades existentes entre grupos sociais no adoecer e morrer.

Tal elucidação é importante no desenho de políticas públicas para que se possa trabalhar com a noção de necessidades diferentes entre distintos grupos sociais, determinadas tanto pela diversidade (sexo, idade, raça) quanto pela injustiça social (renda, acesso a bens e serviços) ou mesmo por características culturais e subjetivas de grupos e indivíduos (Viana, Fausto e Lima, 2003; Travassos, 1997; Tobar, 2003). 
Do ponto de vista da organização dos serviços de saúde no Brasil, a Lei no 8.080 utiliza a noção de "igualdade da assistência à saúde, sem preconceitos ou privilégios de qualquer espécie", para assegurar a utilização equânime dos serviços de saúde. No entanto sabe-se que o texto da lei não é condição suficiente para a garantia do acesso (Brasil, 1990; Duarte, 2000).

De acordo com Duarte (2000), a equidade pode ser examinada sob quatro aspectos:

v equidade ontológica - implica a igualdade fundamental entre as pessoas e aplica-se ao campo dos sistemas morais e religiosos;

v equidade de oportunidade - diz respeito à meritocracia e, como uma característica da doutrina do liberalismo clássico, considera possível a mobilidade social perfeita, a partir da igualdade de oportunidades;

- equidade de condição - não separa equidade de oportunidade uma vez que, para se ter oportunidades iguais, a equidade precisa ser observada pelo "ponto de partida" e deve ser assegurada dando a todos as mesmas condições de vida;

- equidade dos efeitos ou resultados — dá-se quando a legislação e outras medidas políticas procuram compensar os efeitos das desigualdades das condições sociais.

Na prática, a noção de equidade que orienta as políticas desenvolvidas no sistema de saúde brasileiro mescla a equidade de resultados, tendo em vista que as políticas formuladas procuram garantir o acesso das pessoas aos serviços de saúde, com oferta intensiva da atenção primária à população mais pobre com a equidade no cuidado à saúde ao oferecer, para alguns grupos, igualdade de acesso aos serviços para iguais necessidades (Viana, Fausto e Lima, 2003). No caso específico da mamografia, observa-se que os programas e ações formulados estabelecem metas para execução de uma quantidade de exames para um determinado grupo de mulheres por faixa etária, considerando igual necessidade para todas.

A consolidação da equidade na área da saúde consiste, primeiramente, em investir na oferta de serviços para grupos populacionais com acesso e utilização insuficientes, sem reprimir a demanda por serviços necessários a segmentos e grupos que já têm o acesso garantido (Medeiros, 1999).

Em 2004, no estado do Mato Grosso do Sul, o Plano Diretor de Regionalização (PDR) estabeleceu os "Termos de Garantia de Acesso" (documento que contém as metas físicas e orçamentárias das ações a serem ofertadas pelos municípios de referência), incluindo o exame de mamografia para toda a po- 
pulação residente nos municípios do estado, independentemente do local de realização do exame (Mato Grosso do Sul, 2002; Mato Grosso do Sul, Secretaria de Estado de Saúde, 2002).

A revisão da PPI da Assistência foi realizada em 2005, com sua reformulação pelas regiões de saúde e com a participação dos secretários de saúde e técnicos dos municípios, do Grupo de Trabalho da SES e dos auditores da SES/MS dos Núcleos Regionais de Saúde e da Sede, conferindo maior governabilidade aos acordos realizados. A mamografia foi um exame assegurado na programação para toda a população feminina residente no estado.

Neste artigo são discutidos os resultados de uma pesquisa que analisa a cobertura do exame de mamografia no estado de Mato Grosso do Sul na rede pública e a adequação dos encaminhamentos para esse procedimento às recomendações do Programa Nacional de Controle do Câncer do Colo do Útero e de Mama - Viva Mulher - por região de saúde, para estabelecer a relação entre cobertura e equidade no acesso aos serviços.

\section{Metodologia}

O estudo foi realizado exclusivamente na rede pública, com base em dados secundários referentes ao ano de 2004. Foram analisados os 77 municípios do estado de Mato Grosso do Sul. A coleta dos dados e sua análise obedeceram à seguinte classificação: municípios sem serviços de mamografia que encaminham as mulheres para o exame; municípios com serviços de mamografia (municípios-referência) que realizam os exames (em número de quatro: Campo Grande, Dourados, Três Lagoas, Aquidauana); além de Coxim e Corumbá, que tiveram seus dados sobre a produção de mamografias analisados de forma complementar porque, apesar de não serem municípios-referência, possuem equipamentos e realizam exames.

Embora exista consenso quanto à frequência do exame de mamografia bianual a partir dos 40 anos, o exame clínico anual e a ultrassonografia também fazem parte dos exames recomendados a todas as mulheres como forma de acompanhamento e apoio diagnóstico do câncer de mama, mesmo nas faixas etárias em que a mamografia é recomendada. A mamografia é um exame diagnóstico de alta complexidade, conforme a tabela de procedimentos do SUS, que deve ser feito a partir dos 40 anos e a sua indicação, feita por médicos, varia muito e leva em conta diversos fatores.

Ciente da indicação bianual da mamografia para mulheres a partir dos 40 anos, para essa pesquisa foram feitos cálculos para analisar se as necessidades mínimas de realização das mesmas estão sendo supridas no estado, ou 
seja, indicação médica da mamografia após exame clínico, identificação da história da cliente e levantamento de riscos, realização de ultrassonografia de mamas. A metodologia da pesquisa considerou como necessidade mínima a realização da mamografia para $10 \%$ de mulheres na faixa de idade entre 40 49 anos e bianual para mulheres na faixa de idade entre 50-69 anos. O cálculo ficou assim definido: $10 \%$ do total de mulheres entre 40-49 anos residentes no município $+50 \%$ das mulheres de 50-69 anos (Brasil, Ministério da Saúde, 2002; Brasil, Ministério da Saúde, Instituto Nacional do Câncer, 2005).

Os dados da população por faixa etária foram extraídos do Datasus, com base nas estimativas do IBGE para 2004 (Ministério da Saúde, Datasus, 2006). Os dados de produção dos exames de mamografia, referentes aos meses de janeiro a dezembro de 2004, foram coletados junto às secretarias municipais de saúde de Campo Grande, Aquidauana, Dourados e Três Lagoas (municípiosreferência), e Coxim e Corumbá que, embora não sejam municípios-referência, realizaram exames em 2004.

Foram utilizados como fontes documentais: o termo de garantia de acesso assinado pelos gestores na PPI da assistência; o sistema de informações ambulatoriais (SIA/SUS) referente à produção pelas unidades de saúde do exame de mamografia; os projetos diretrizes "Câncer de Mama - Prevenção Secundária", da Sociedade Brasileira de Mastologia e da Federação Brasileira das Sociedades de Ginecologia e Obstetrícia; o documento de consenso para o câncer de mama.

A verificação da ocorrência do exame de mamografia deu-se com o preenchimento de um instrumento e com a análise dos livros de registros existentes nos serviços que executam o procedimento nos seis municípios, que continham os seguintes dados: data, nome da cliente e município de residência, embora não existam normas para o registro do procedimento. Quanto aos municípios que encaminham as solicitações, com algumas exceções, não há padronização desses dados.

$\mathrm{O}$ instrumento de pesquisa, preenchido para cada município, era dividido em três partes. A primeira com o número de unidades, mamógrafos e profissionais cadastrados nos seis municípios que realizam o exame. A segunda com as informações referentes à programação no que diz respeito à abrangência da região quanto ao número de mulheres nas faixas etárias pesquisadas, os municípios pertencentes à região e número de exames programados. Na terceira, a verificação dos livros de registros existentes em cada unidade credenciada e a contagem do número de exames realizados, considerando o município de residência da cliente. Os dados foram comparados ao número de exames necessários de acordo com a população-alvo e com o número de exames programados conforme os termos de compromisso de acesso pactuados na PPI. 
$\mathrm{Na}$ análise, dois aspectos foram fundamentais para conhecer as características do acesso ao exame de mamografia: com os dados referentes ao número de exames realizados por região do estado e por residência da paciente foi possível conhecer o número de exames realizados em todo o território estadual e verificar a diferença de cobertura entre os municípios de residência e os municípios-referência; os dados relativos aos valores pactuados na PPI para 2004 foram comparados à produção de mamografia no mesmo período.

\section{Resultados}

O PDR ao ser elaborado em Mato Grosso do Sul teve como objetivo criar sistemas funcionais de saúde em que os cidadãos tivessem acesso garantido a todas as ações e serviços para o atendimento de suas necessidades - o mais próximo possível do seu local de moradia para ações de menor complexidade, e em quaisquer outros níveis dentro e fora do estado para ações de complexidade e custo maiores. Procurando atender aos preceitos da regionalização estabelecidos na Noas 2002, Mato Grosso do Sul foi dividido em três grandes regiões, 11 microrregiões e 23 módulos assistenciais (Mato Grosso do Sul, 2002). Inicialmente, a PPI da assistência fez a programação com base principalmente na série histórica de realização de procedimentos. Além disso, utilizou os parâmetros da Consulta Pública SAS/MS no 1, de 8 de dezembro de 2000 (Brasil, Ministério da Saúde, 2002).

Em 2004, Mato Grosso do Sul tinha uma população aproximada de 2,2 milhões de habitantes. Destes, 249.708 mulheres estavam na faixa etária de 40 a 69 anos, com uma necessidade estimada de 74.712 exames de mamografia. A Portaria no 1.101/GM (Ministério da Saúde, 2002) define como parâmetro a instalação de um mamógrafo para cada 240 mil habitantes, e o estado conta com 10 aparelhos de mamografia credenciados com capacidade para realizar em torno de 89 mil exames anualmente, número suficiente previsto pelo Consenso, que era de 74.712 exames.

Os 10 mamógrafos que atendem a rede pública estavam assim distribuídos: um em Aquidauana (755 exames); cinco em Campo Grande (17.361 exames); um em Coxim (99 exames); dois em Dourados (3.310 exames) e um em Três Lagoas (1.723 exames). O total de exames de mamografia em 2004 foi de 23.248, corroborando as informações do SIA/SUS.

$\mathrm{Na}$ época, o exame de mamografia era agendado no serviço de saúde do município de origem da usuária para ser realizado no serviço credenciado do município-referência. O município de Campo Grande, capital do estado, foi o 
único município-referência que não agendou a mamografia para clientes de outros municípios, a despeito de ter assinado os termos de garantia de acesso. No entanto, de acordo com os registros de produção, realizou a mamografia em 319 não residentes.

O estado é subdividido em três regiões assistenciais:

v região de Campo Grande - com cinco microrregiões, nove módulos assistenciais e 33 municípios. É a maior região do estado, com 65,8\% da sua área total nas porções central, setentrional e ocidental, com uma população de 654.934 mulheres. Realizou 16.979 exames de mamografia para uma necessidade de 44.703 exames. Ficou com cobertura em torno de $37,98 \%$, a maior do estado. Do ponto de vista da produção, realizou $78,3 \%$ de todos os exames de mamografia feitos no estado;

v região de Dourados - com quatro microrregiões, nove módulos assistenciais e 34 municípios. Está localizada na porção meridional do estado, ocupando uma área correspondente a 18,35\% do território estadual e tem uma população de 327.660 mulheres. Realizou 4.003 exames de mamografia para uma necessidade de 21.621 exames, ficando com uma cobertura de $18,51 \%$. A região foi responsável por $14,2 \%$ dos exames de mamografia realizados no estado;

、 região de Três Lagoas - é a menor região, com duas microrregiões, cinco módulos assistenciais e 10 municípios. Está localizada no extremo oriental, ocupando $15,8 \%$ da área do estado, com aproximadamente 215.719 mulheres. A região alcançou uma cobertura de $22,52 \%$ (1.889 exames), bem abaixo dos 8.388 necessários, executando 7,4\% de todos os exames de mamografia em Mato Grosso do Sul.

Ao observar o número de exames realizados em cada município, conforme exemplos citados na tabela 1, são identificadas grandes diferenças no número de exames realizados conforme município de origem da mulher. Apesar da insuficiência no número de exames em geral no estado, enquanto em alguns municípios a cobertura fica em torno de $50 \%$ da quantidade necessária, em outros a cobertura não alcança $10 \%$.

O município de Campo Grande concentra o maior número de mamógrafos do estado e é referência para grande parte da sua região, exceto para os municípios em torno de Aquidauana (Anastácio, Bodoquena, Dois Irmãos do Buriti, Miranda, Nioaque). Apresenta para a população residente uma cobertura de $56,75 \%$ de exames, superior em relação aos demais municípios da 
região. A região de Campo Grande é a que mais apresenta desigualdades de acesso à mamografia, pois a cobertura de exames varia de $0,20 \%$ para o município de Ladário até $56,75 \%$ para a população residente na capital. Uma das possíveis causas é a extensão territorial, além da dificuldade de acesso, já que há municípios que estão distantes mais de $400 \mathrm{~km}$ da capital, como Porto Murtinho, Corumbá e Ladário. A região de Três Lagoas é a que apresenta maior igualdade no acesso ao exame. O número mais baixo de exames realizados foi em Aparecida do Taboado, com cobertura de $16,91 \%$, e o mais alto em Água Clara, com cobertura de $43,57 \%$.

\section{Tabela 1}

Principais diferenças de cobertura de exames nas três regiões assistenciais do estado em 2004

\begin{tabular}{|c|c|c|c|c|}
\hline Região & $\begin{array}{l}\text { Municípios com maior e } \\
\text { com menor cobertura }\end{array}$ & $\begin{array}{c}\text { Número } \\
\text { de exames } \\
\text { necessários }\end{array}$ & $\begin{array}{c}\text { Número de } \\
\text { exames realizados }\end{array}$ & $\begin{array}{c}\% \text { de } \\
\text { cobertura }\end{array}$ \\
\hline \multirow[t]{3}{*}{ Campo Grande } & & & & 37,98 \\
\hline & Campo Grande & 26.261 & 14.903 & 56,75 \\
\hline & Guia Lopes da Laguna & 441 & 1 & 0,23 \\
\hline \multirow[t]{3}{*}{ Dourados } & & & & 18,51 \\
\hline & Mundo Novo & 588 & 281 & 47,79 \\
\hline & Japorã & 173 & 2 & 1,16 \\
\hline \multirow[t]{3}{*}{ Três Lagoas } & & & & 22,52 \\
\hline & Água Clara & 241 & 105 & 43,57 \\
\hline & Aparecida do Taboado & 822 & 139 & 16,91 \\
\hline Mato Grosso do Sul & & 74.712 & 23.248 & 32,03 \\
\hline
\end{tabular}

Obs.: o número de exames necessários e realizados expressa a ocorrência nos municípios citados na segunda coluna.

Em média, a cobertura de exames de mamografia realizados em Mato Grosso do Sul pelo SUS foi de 32,03\%, incluídos os exames em que não registraram o local de residência da cliente. No estado, há equipamentos em número suficiente para atender a toda a população; no entanto isso não garante a produção dos serviços, pois depende também da existência de número adequado de profissionais, de materiais e de mecanismos administrativos para garantir a utilização dos serviços.

Pode-se observar na figura a evolução da produção de exames de mamografia entre 2000 e 2004 em Mato Grosso do Sul, com um crescimento 
acima de $200 \%$, enquanto a população feminina entre 40 e 69 anos, que era de 235.640 mulheres em 2000, até 2004 sofreu um aumento de aproximadamente $5,6 \%$.

Produção de mamografias em Mato Grosso do Sul, de 2000 a 2004

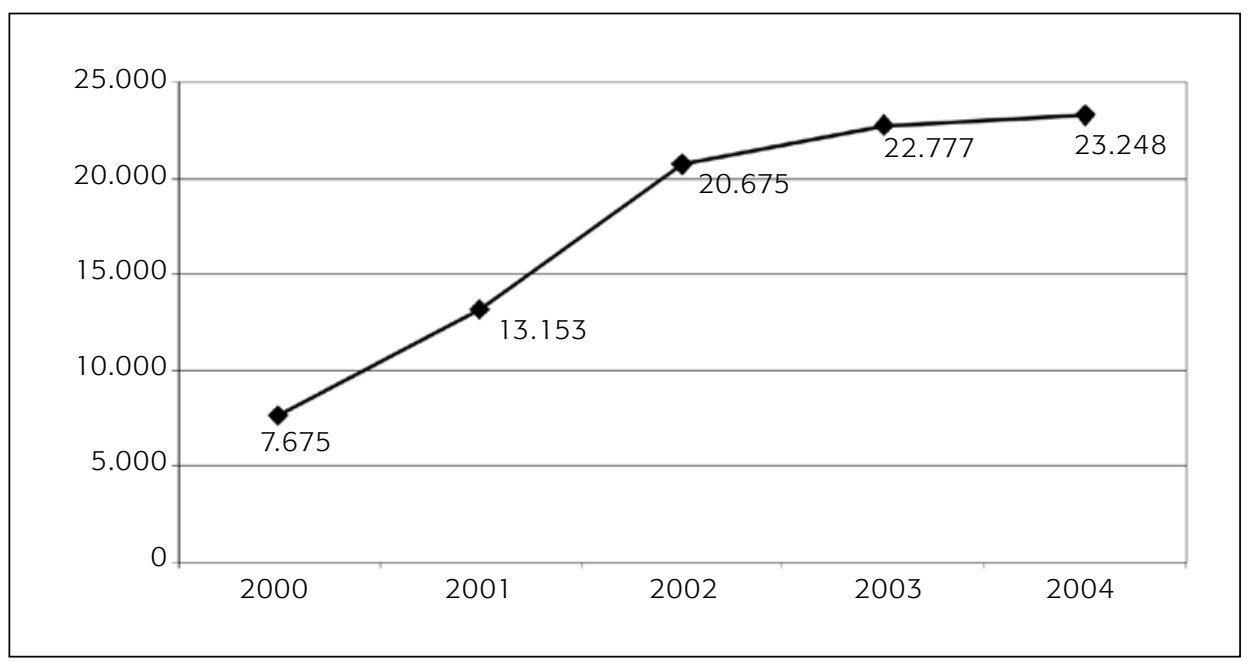

Fonte: SIA/SUS - Datasus.

A tabela 2 representa o comparativo entre o que foi programado para execução dos termos de garantia de acesso nas microrregiões de cada região assistencial, o realizado e a necessidade mínima de exames de mamografia para a população feminina na faixa etária de 40-69 anos, em que o número de exames necessários para essa faixa etária é maior do que a quantidade programada na PPI da assistência de 2002.

São observadas discrepâncias entre o número de exames necessários, programados e realizados. É possível, então, fazer algumas ponderações: o número de exames programados obedeceu a uma série histórica que não atendia às necessidades. As recomendações para dimensionar a quantidade necessária com base nas informações demográficas não foram consideradas; a figura indica aumento constante no número de exames realizados sem considerar a quantidade programada, o que sugere que os profissionais e a população estão sensibilizados para o agravo e buscando diagnóstico precoce. Assim, o aumento da demanda e a realização crescente de exames além do programado tende a induzir a uma revisão das quantidades pactuadas e melhor adequação da oferta. 
Tabela 2

Demonstrativo do número de mamografias programadas

na PPI e realizadas para a faixa etária 40-69 anos em 2004 para as microrregiões do estado

\begin{tabular}{|llccc|}
\hline Região & Microrregião & $\begin{array}{c}\text { № de exames } \\
\text { programados na PPI }\end{array}$ & $\begin{array}{c}\text { № de exames } \\
\text { realizados }\end{array}$ & $\begin{array}{c}\text { № mínimo de } \\
\text { exames necessários }\end{array}$ \\
\hline Campo Grande & Aquidauana & 545 & 800 & 4.146 \\
& Campo Grande & 377 & 15.671 & 31.345 \\
& Corumbá & 494 & 244 & 3.835 \\
& Coxim & 317 & 162 & 2.301 \\
& Guia Lopes & 400 & 102 & 3.076 \\
Dourados & Dourados & 1.328 & 2.624 & 10.433 \\
& Naviraí & 463 & 669 & 3.383 \\
& Nova Andradina & 410 & 381 & 3.255 \\
& Ponta Porã & 637 & 329 & 4.550 \\
Três Lagoas & Paranaíba & 377 & 697 & 3.522 \\
& Três Lagoas & 595 & 1.192 & 4.866 \\
\hline
\end{tabular}

Obs.: o número de exames programados e realizados expressa a ocorrência de exames nas microrregiões citadas na segunda coluna.

\section{Discussão}

Foram muitas as dificuldades para a realização do estudo que originou este artigo. Destaca-se a ausência ou a diversidade na forma de registro dos procedimentos nos municípios-referência. $\mathrm{O}$ principal entrave foi referente à falibilidade dos dados do SIA/SUS, que, por se tratar de um sistema nacional, deveria possuir informações cruciais como: faixa etária; município de referência; e grupo de atendimento. Dada essa dificuldade, os dados foram coletados diretamente nos livros de registros das unidades que realizam o procedimento e, mesmo assim, observou-se falta de registros sistematizados e monitorados, diminuindo a qualidade dos dados.

À época da elaboração da PPI ficou definido que a programação deveria ser orientada pelos parâmetros da Portaria oㅜ 1.101 do Ministério da Saúde (2002) e pelas séries históricas de utilização dos serviços em cada município. Os termos de garantia de acesso se basearam quase exclusivamente em registros anteriores de procedimentos realizados nos municípios, aumentando a 
possibilidade de agravamento de algumas desigualdades, pois nos municípios em que não há oferta, não há registro de ação realizada.

Algumas das diretrizes que estavam previstas na definição de mecanismos e instrumentos para regulação da assistência à saúde não foram implantadas, como: acompanhamento do cumprimento dos termos de garantia de acesso; subsídio aos gestores municipais para regulação do fluxo de pacientes em consequência dos compromissos firmados; formação de quadros de recursos humanos da Secretaria de Estado de Saúde e dos municípios para implantação e manutenção das ações de regulação da assistência à saúde; criação de um sistema de informações com dados sobre a capacidade instalada, a produção de serviços, as necessidades e a programação da assistência, a distribuição espacial dos serviços no estado para embasar a contratação de novos serviços e a revisão da programação da assistência (Mato Grosso do Sul, Secretaria de Estado de Saúde, 2002).

Com a definição de metas para o controle do câncer de mama, que busca um aumento de $60 \%$ na realização de exames de mamografia e com base na discussão e resultados apresentados na pesquisa, seria importante discutir para o estado do Mato Grosso do Sul quais as metas e a programação pactuada, bem como os parâmetros necessários ao efetivo controle do câncer de mama. Para tanto, seria preciso desenvolver um sistema de informações que permitisse monitorar e avaliar essas metas, compatibilizando-as à integração regional e ao cumprimento das políticas planejadas, financiadas e executadas (Travassos e colaboradores, 2000; Lucchese, 2003).

Aspectos elementares como a ausência de registro de atendimento e de faixa etária no sistema de informações SIA/SUS são importantes fatores que não permitiram o acompanhamento dos resultados. Quando fornecidos, são relevantes para identificação dos níveis de cobertura e de acesso, e estes podem subsidiar as decisões no processo de gestão regional e interregional.

Os dados aqui discutidos e analisados apontam para um aumento importante na produção de exames de mamografia que, no entanto, ainda não são suficientes para atender à demanda em Mato Grosso do Sul. As diferenças observadas em relação ao que foi programado, o realizado e o necessário mostram a necessidade de revisar as metas e de que a discussão leve em conta a série histórica, o perfil epidemiológico e os parâmetros definidos nos documentos técnicos.

Se observada a cobertura nas regiões e por municípios, constata-se que a PPI e o PDR não conseguiram garantir a igualdade de acesso da população 
feminina ao exame de mamografia, muito provavelmente em decorrência da: não implantação dos mecanismos de regulação e efetivação na íntegra quanto aos pactos estabelecidos para garantia do acesso; falta de financiamento compatível com o planejamento da PPI; baixa governabilidade do órgão estadual; fragmentação ou inexistência de responsabilidades atribuídas; descompasso entre as programações pactuadas e comportamento das populações. Podese também dizer que a equidade não foi garantida. Nesse sentido, Lucchese (2003) e Travassos (1997) discutem que o alcance da equidade depende da conjugação de fatores que vão de um sistema de financiamento estável com vistas ao fomento da equidade inter-regional até um sistema de gestão flexível para dispor dos recursos humanos, financeiros e tecnológicos com a responsabilização dos gestores pelos resultados.

\section{Conclusões}

Existem grandes diferenças de cobertura entre todos os municípios de Mato Grosso do Sul, o que indiretamente sugere desigualdade no acesso das mulheres ao exame de mamografia. A região que concentra maior número de equipamentos apresentou as maiores diferenças no acesso das mulheres ao exame. Para um estado que no ano da realização da pesquisa teve 104 mortes em decorrência de câncer de mama e apresenta os mais altos índices de incidência do Brasil, os diferentes resultados de cobertura para os diferentes municípios do estado sugerem a necessidade de discutir e propor estratégias mais equânimes de acesso.

\section{Referências}

BRASIL. Ministério da Saúde. Lei no 8.080, de 19 de setembro de 1990. Dispõe sobre as condições para a promoção da saúde, a organização e o funcionamento dos serviços correspondentes. Diário Oficial da União, Brasília, 1990.

. Ministério da Saúde. Portaria no 1.101/GM, de 12 de junho de 2002. Estabelece os parâmetros de cobertura assistencial no âmbito do Sistema Único de Saúde (SUS). Brasília, 2002.

. Ministério da Saúde. Instituto Nacional do Câncer. Programa Nacional de Controle do Câncer do Colo do Útero e de Mama - Viva Mulher. Brasília, 2005. Disponível em: < www.inca.gov.br/conteudo_view.asp?id=140 >. 
Ministério da Saúde. Controle do câncer de mama: documento de consenso. Rio de Janeiro, 2004. Disponível em: <www.inca.gov.br/publicacoes/consensointegra.pdf $>$.

. Ministério da Saúde. Plano de ação para o controle dos cânceres do colo do útero e da mama 2005-2007. Brasília, 2005.

. Ministério da Saúde. Secretaria de Assistência à Saúde. Departamento de Regulação, Avaliação e Controle de Sistemas. Diretrizes para a programação pactuada e integrada da assistência à saúde. Brasília, 2006.

. Ministério da Saúde. Portaria no 399/GM/Ministério da Saúde, de 22 de fevereiro de 2006. Divulga o Pacto pela Saúde 2006 - consolidação do SUS e aprova as diretrizes operacionais do referido pacto. Brasília, 2006.

DUARTE, C. M. R. Equidade na legislação: um princípio do sistema de saúde brasileiro? Ciência \& Saúde Coletiva, v. 5, n. 2, p. 443-463, 2000.

KEMP , C. et al. Câncer de mama - prevenção secundária. In: ASSOCIAÇÃO MÉDICA BRASILEIRA E CONSELHO FEDERAL DE MEDICINA. Projeto Diretrizes 2002. Rio de Janeiro, 2002. Disponível em: <www.sbmastologia.com.br/upload/ sbm/conheca/projeto_diretrizes3.pdf $>$.

LUCCHESE, P. T. R. Equidade na gestão descentralizada do SUS: desafios para a redução de desigualdades em saúde. Ciência \& Saúde Coletiva, v. 8, n. 2, p. 439448, 2003.

MATO GROSSO DO SUL. PDR/Plano Diretor de Regionalização da Assistência à Saúde. Campo Grande, 2002. Disponível em: <www.saude.ms.gov.br/externo/ downloads/PDR.zip >.

. Secretaria de Estado de Saúde. Resolução no 440/02/SES/MS, de 12 de agosto de 2002. Aprova as decisões da Comissão Intergestores Bipartite Estadual. Diário Oficial do Estado de Mato Grosso do Sul, Campo Grande, 2002.

MEDEIROS, M. Princípios de justiça na alocação de recursos em saúde. Rio de Janeiro: Ipea, 1999. (Textos para Discussão).

MINISTÉRIO DA SAÚDE. Datasus. Informações em saúde. Demográficas e Socioeconômicas. Brasília, 2006. Disponível em: < http://tabnet.datasus.gov.br/cgi/tabcgi. exe?ibge/cnv/popms.htm>.

TOBAR, F. et al. Modelos de equitativos de distribución de recursos sanitarios. Washington, 2003. Disponível em: <www.opas.org.br/servico/arquivos/Sala5420. pdf $>$.

TRAVASSOS, C. Equidade e o Sistema Único de Saúde: uma contribuição para debate. Cadernos de Saúde Pública, v. 13, n. 2, p. 325-330, 1997. 
et al. Desigualdades geográficas e sociais na utilização de serviços de saúde no Brasil. Ciência \& Saúde Coletiva, v. 5, p.133-149, 2000.

VIANA, A. L.; FAUSTO, M. C. R.; LIMA, L. D. Política de saúde e equidade. São Paulo em Perspectiva, v. 17, n. 1, p. 58-68, 2003.

WHITEHEAD, M. The concepts and principles of equity and health. Copenhagen: World Health Organization Regional Office for Europe, 2000. 